\title{
Laterality and unilateral deafness: Patients with congenital right ear deafness do not develop atypical language dominance
}

\author{
Lise Van der Haegen ${ }^{1}$, Frederic Acke ${ }^{2}$, Guy Vingerhoets ${ }^{1}$, Ingeborg Dhooge ${ }^{2}$, Els De Leenheer ${ }^{2}$, Qing \\ $\mathrm{Cai}^{3}$, Marc Brysbaert ${ }^{1}$ \\ 1 Department of Experimental Psychology, Ghent University, Belgium \\ 2 Department of Otorhinolaryngology, Ghent University Hospital, Ghent, Belgium \\ 3 Key Laboratory of Brain Functional Genomics, Ministry of Education, Shanghai, Key Laboratory of \\ Brain Functional Genomics, Institute of Cognitive Neuroscience, East China Normal University, China
}

Address for Correspondence:

Qing Cai

Institute of Cognitive Neuroscience,

School of Psychology and Cognitive Sciences,

East China Normal University,

Shanghai 200062, China.

Email: qcai@psy.ecnu.edu.cn

Tel: (+86)-21-62233280 


\begin{abstract}
Auditory speech perception, speech production and reading lateralize to the left hemisphere in the majority of healthy right-handers. In this study, we investigated to what extent sensory input underlies the side of language dominance. We measured the lateralization of the three core subprocesses of language in patients who had profound hearing loss in the right ear from birth and in matched control subjects. They took part in a semantic decision listening task involving speech and sound stimuli (auditory perception), a word generation task (speech production) and a passive reading task (reading). The results show that a lack of sensory auditory input on the right side, which is strongly connected to the contralateral left hemisphere, does not lead to atypical lateralization of speech perception. Speech production and reading were also typically left lateralized in all but one patient, contradicting previous small scale studies. Other factors such as genetic constraints presumably overrule the role of sensory input in the development of (a)typical language lateralization.
\end{abstract}

Keywords: Hemispheric asymmetry; deafness; speech perception; speech production; reading 


\section{Introduction}

Language is well-known to be lateralized in humans. Numerous studies have reported a dominance of the left hemisphere for speech production, auditory perception, and reading (see Price, 2012 for a review). These three core subprocesses of language are the focus of the current study.

Speech production mainly activates the left middle and inferior frontal gyrus (IFG) or the so-called Broca's area including the pars opercularis and pars triangularis. Activity extends to other areas such as the cerebellum for the fast temporal organization of speech, the ventral premotor area for articulatory planning, pre- and post-central motor regions associated with mouth movements, the superior temporal gyri (STG)/sulci (STS) and planum temporale involved in auditory feedback.

Perception of speech relative to non-speech has been related to the left anterior and posterior STG/STS (aSTS/pSTS) surrounding the transverse gyrus of Heschl, to the left IFG and premotor areas for articulatory recoding and the attentional ventral supramarginal gyrus. When semantic comprehension is involved, the activity in the aSTS and pSTS is more widespread, in addition to for example the angular gyri for narrative comprehension. The pathway that auditory stimuli follow from the ear to the human cortex is complex due to parallel and crossed fiber tracts, but more nerve fibers lead to contralateral than ipsilateral brain areas. The left auditory cortices have been found to be specialized in fast temporally changing stimuli such as in speech, whereas the right homologue areas are found to be dominantly involved in tonal information processing (Firszt, Ulmer \& Gaggl, 2006).

Finally, reading has been related to the left ventral occipito-temporal (vOT) region therefore called the visual word form area (Cohen et al., 2000). The exact nature of the region is still under debate, but the anterior part has been related to phonological and lexico-semantic processes of reading, whereas the posterior part is more responsible for visual features (Seghier \& Price, 2011). Reading requires bilateral visual input. Due to the partial crossing of optic fibers, left/right visual field information is initially projected to the right $(\mathrm{RH}) / \mathrm{left}(\mathrm{LH})$ hemisphere 
respectively. The information is however thought to be early reunited in the dominant LH before reading proper starts (Van der Haegen \& Brysbaert, 2011).

The origins of hemispheric specialization have been attributed to several influences such as genetic, evolutionary, developmental and environmental factors (Bishop, 2013; Hervé, Zago, Petit, Mazoyer \& Tzourio-Mazoyer, 2013). Pinel et al. (2014) compared the correlations between monozygotic and dizygotic twins in vOT lateralization during word reading, and found evidence for a partial genetic influence. Genetic influences are often associated with anatomical asymmetries, such as a deeper RH than LH pit in the STS found in both young infants and adults (Leroy et al., 2015). Greve et al. (2013) found significant differences in the surface area of the STG and vOT when comparing left-handers with LH and RH speech dominance. In Vingerhoets et al. (2013), correlations between the side and degree of praxis and speech lateralization pointed to a common evolutionary origin. Finally, (developing) higher-order cognitive functions can influence each other's asymmetry. For example, learning how to read leads to a LH lateralization in the occipitotemporal cortex which in turn may force face lateralization to be dominantly processed in the homologue area in the RH (Cantlon et al., 2011; Behrmann \& Plaut, 2015). In adults, lateralization indices correlate between reading and speech production (Van der Haegen, Cai, Brysbaert, 2012) and between reading and speech comprehension (Pinel et al., 2014). The purpose of this study is to test another possible environmental influence on the lateralization of speech production, reading and speech comprehension, namely sensory deprivation and more specifically a lack of sensory auditory input in congenital unilateral deaf patients.

Previous studies already described neural adaptions in sensory deprived subjects. The usual finding is that patients with unilateral hearing loss show more activity in the ipsilateral hemisphere upon hearing stimuli in the intact ear, suggesting some type of plasticity in brain functioning (e.g. Burton, Firszt, Holden, Agato, \& Ushanski, 2012 who presented noise-like random spectrogram sounds to left or right ear unilateral deaf patients who had developed profound hearing loss after birth, most often diagnosed after language development. Subjects performed an fMRI odd-ball task in which they had to press a button when hearing a deviant stimulus). In normally hearing participants, the contralateral hemisphere is stimulated more than the ipsilateral when auditory stimuli are presented unilaterally, in line with the typical dominance 
of crossed projections over uncrossed. In patients with unilateral hearing loss, however, the ipsilateral projections seem to gain importance. The difference is not always found, however (e.g., in an EEG study by Hine, Thornton, Davis, \& Debener, 2008, with tone and noise stimuli while subjects watched a silent movie), raising questions about the magnitude and practical importance of the finding. Jensen et al. (1989) in addition proposed the right-ear advantage hypothesis, according to which unilateral left ear deafness would have less detrimental effects on cognitive performance than unilateral right ear deafness, because in the former case the contralateral connections to the language dominant hemisphere are still intact. Their conclusion was based on better recognition of interrupted speech in background noise for left ear compared to right ear hearing impaired children.

Two factors are likely to have an effect on the laterality findings in patients with sensory deficits. First, it can be expected that the effects will be larger in patients with congenital deprivation than in patients who acquired sensory deprivation later in life. For instance, Gordon, Wong, and Papsin (2013) argued that congenitally deaf children better get bilateral cochlear implants, because a unilateral implant may cause permanent reorganization of the brain. They presented evidence from an EEG study measuring cortical activity during tone listening that unilateral implants may overactivate the contralateral hemisphere due to the lack of inhibition from the deaf ear. So, whereas later acquired unilateral deafness seems to result in strengthening the ipsilateral pathway (Burton et al., 2012), congenital unilateral deafness may lead to overexcitation of the contralateral pathway. The second factor that is likely to have an effect is the degree of hearing loss. One can optimize the clarity of the findings about lateralization in patients with sensory deprivation by limiting the study to participants with profound unilateral hearing loss (at least with respect to speech-related stimuli, so that no verbal input enters the brain via the affected ear).

One study followed the above two criteria (Adorni et al., 2013). An additional appeal of the study was that it investigated language lateralization rather than responses to low-level auditory stimuli. Reading lateralization was examined in a 31-year old female patient, RA, who was congenitally deaf in the right ear. She performed a letter detection task while event-related potentials were recorded. By comparing the N170 to words and non-orthographic control stimuli, 
Adorni et al. (2013) concluded that the visual word form area in this patient was situated in the right hemisphere, and not in the left hemisphere as seen in all the control participants tested with the same paradigm. Whereas the normalized hemispheric difference lateralization index based on the amplitudes of temporal occipital electrodes was +0.33 for the control participants, it was 0.47 for RA. Adorni et al. ventured that the atypically lateralized vOT might be due to the fact that auditory word processing in the patient is also lateralized to the right hemisphere, as a result of the congenital lack of input from the right ear. However, the authors did not test the laterality of auditory word recognition in RA and one should be careful not to draw strong conclusions on the basis of a single case study. Finding a higher chance of developing an atypical dominance for speech production, reading and auditory perception in a larger sample without input from the right ear would question a strong genetic origin of language dominance and would also provide further evidence for the warning that the complete absence of input from one ear may increase the strength of the contralateral pathway of the other ear (Gordon et al., 2013).

To investigate the issue properly, we searched for a reasonably large group of persons with profound, congenital, unilateral hearing loss in the right ear, and compared them to a control group. We also tested all three main language functions: speech production, speech perception, and word reading. Finally, we used paradigms that have shown a robust left hemispheric dominance in previous studies. These were a word generation task for speech production (Van der Haegen, Cai, Seurinck \& Brysbaert, 2011), an auditory semantic decision task to evaluate speech perception (Thierry, Giraud \& Price, 2003), and a passive reading task to test reading lateralization (Cohen et al., 2002). We used fMRI paradigms to give us detailed spatial information.

\section{Method}

\section{$2.1 \quad$ Participants}

Participants' inclusion criteria were the presence of a congenital profound unilateral right-sided hearing impairment, and age between 18-70y. Exclusion criteria were any significant neurological or psychiatric disorder, the presence of left-sided hearing impairment, and contraindications for fMRI testing. Seven patients were willing to take part in the study. This size is sufficient to test the strong claim made by Adorni et al. (2013) and also to find clinically 
meaningful increases in the probability of atypical brain dominance. The prevalence of newborns with congenital unilateral hearing loss is estimated to be 2-4 per 1000 in the US (White, 2004). Since 1998 standard, universal, neonatal hearing screening has been implemented in Flanders. Demographic data and etiology of the patients' deafness can be found in Table 1. Seven control participants matched on sex, age and education level (i.e. having a degree in higher education or not) were added for comparison purpose.

TABLE 1. Demographic data of participants include: Participant number, initials, sex and age. Handedness scores are Edinburgh Handedness Index (EHI), finger tapping index, familial sinistrality (i.e. number of left-handed/total number of first-degree family members). Cognitive performance was tested by the reading tests One-Minute-Test (OMT) and Klepel and general executive function test Symbol digit modality test (SDMT). Hearing performance is expressed as pure tone average (PTA, expressed in $\mathrm{dB}$ HL; >120 corresponds with no detectable thresholds at maximal intensity) thresholds for the left and right ear, and finally the etiology of the right ear deafness if known. Control subjects are highlighted in grey.

\begin{tabular}{|c|c|c|c|c|c|c|c|c|c|c|c|c|}
\hline $\mathrm{Nr}$ & Initials & Sex & Age & $\mathrm{EHI}$ & $\begin{array}{l}\text { Finger } \\
\text { tapping }\end{array}$ & $\begin{array}{c}\text { Fam. } \\
\text { Sinistrality }\end{array}$ & Wordgen & $\begin{array}{l}\text { OMT/ } \\
\text { Klepel }\end{array}$ & SDMT & $\begin{array}{l}\text { PTA } \\
\text { left } \\
\text { ear }\end{array}$ & $\begin{array}{c}\text { PTA } \\
\text { right } \\
\text { ear }\end{array}$ & $\begin{array}{c}\text { Subjective } \\
\text { etiology }\end{array}$ \\
\hline 1 & WV & $M$ & 29 & 100 & 0.2 & $1 / 4$ & $9.7(2.7)$ & $103 / 90$ & 48 & -2 & $>120$ & $\begin{array}{c}\text { aplastic } \\
\text { cochlear nerve }\end{array}$ \\
\hline 2 & MS & $\mathrm{F}$ & 59 & 74 & 4.8 & $0 / 10$ & $6.2(1.5)$ & $112 / 108$ & 69 & 10 & $>120$ & unknown \\
\hline 3 & ND & $\mathrm{F}$ & 41 & 100 & -1.6 & $0 / 6$ & $7.1(2.0)$ & $116 / 110$ & 49 & -2 & $>120$ & unknown \\
\hline 4 & $\mathrm{AF}$ & $M$ & 32 & 92 & 13.0 & $1 / 3$ & $5.8(2.5)$ & $116 / 111$ & 66 & -2 & $>120$ & absent cochlea \\
\hline 5 & $\mathrm{ML}$ & $\mathrm{F}$ & 69 & 64 & 9.9 & $0 / 7$ & $8.6(1.1)$ & $104 / 110$ & 46 & 15 & $>120$ & unknown \\
\hline 6 & NV & $\mathrm{F}$ & 36 & 92 & 12.7 & $0 / 5$ & 7.7 (1.9) & $116 / 107$ & 72 & 2 & $>120$ & unknown \\
\hline 7 & KW & $\mathrm{F}$ & 49 & 100 & 0.7 & $2 / 6$ & $9.4(1.8)$ & $102 / 107$ & 64 & 3 & $>120$ & $\begin{array}{c}\text { aplastic } \\
\text { cochlear nerve }\end{array}$ \\
\hline 8 & MV & $M$ & 31 & 81 & 2.9 & $3 / 4$ & $4.2(1.0)$ & $109 / 100$ & 61 & 0 & 0 & NA \\
\hline 9 & $A B$ & $\mathrm{~F}$ & 59 & 74 & 0.2 & $0 / 8$ & $7.2(2.3)$ & $91 / 89$ & 55 & 8 & 12 & NA \\
\hline 10 & ED & $\mathrm{F}$ & 41 & 91 & 4.9 & $1 / 7$ & $7.1(2.5)$ & $116 / 114$ & 60 & 12 & 12 & NA \\
\hline 11 & MT & $M$ & 31 & 73 & 4.8 & $0 / 3$ & $6.9(2.0)$ & $114 / 97$ & 75 & -5 & -3 & NA \\
\hline 12 & RV & $F$ & 70 & 91 & 23.0 & $1 / 10$ & $5.9(1.3)$ & $78 / 73$ & 72 & 15 & 10 & NA \\
\hline 13 & $\mathrm{RL}$ & $\mathrm{F}$ & 38 & 100 & 0.4 & $0 / 2$ & $9.4(2.8)$ & $116 / 114$ & 79 & -3 & 0 & NA \\
\hline 14 & $\mathrm{EH}$ & $\mathrm{F}$ & 47 & 100 & 4.3 & $2 / 7$ & $8.2(2.9)$ & $101 / 110$ & 69 & 7 & 2 & NA \\
\hline
\end{tabular}

For each participant we assessed their lateral preferences index for handedness (Edinburgh Handedness Inventory), footedness and eyedness (Oldfield, 1971). Indices were calculated as $(\mathrm{RH}-\mathrm{LH} / \mathrm{RH}+\mathrm{LH}) * 100$. All participants were right-handed, reducing the a priori chances of right hemisphere language dominance to less than 5\% per participant (Knecht et al., 2000; Loring et al., 1990). In addition, performance of handedness was measured by a finger tapping task, in which participants had to press a button as many times as possible within 10 
seconds. Five blocks were tested for each hand, starting with the index finger of their dominant hand. They were asked to keep their wrist on the table to ensure the same hand position for their left and right hand. An index was calculated in the same way as the lateral preference index, with $\mathrm{RH} / \mathrm{LH}$ being the average number of button presses by the right and left hand respectively (see Table 1 for individual scores). All but one participant showed a better performance with their right hand, although the hand differences were often small. Finally, familial sinistrality was measured by asking participants to report the handedness of their parents, children and siblings.

Two reading tests were run to exclude reading disorders. In the One Minute Test (Brus \& Voeten, 1991), participants are asked to read out loud as many words as possible within one minute. Stimuli consist of 116 words with increasing reading difficulty. The Klepel (van den Bos, Spelberg, Scheepsma \& de Vries, 1999) is a similar test with pseudowords. Scores of both tests (i.e. total number of words read correctly) can be found in Table 1. Scores were comparable for patients and control subjects (One Minute Test: Mean: 110, SD: 6.6 for patients and Mean: 104, SD: 14.5 for controls; Klepel: Mean: 106, SD: 7.3 for patients and Mean: 100, SD: 15.0 for controls). Finally, the Symbol digit modalities test (Smith, 1973) confirmed that all patients and control participants had normal general executive functioning. In this paper-and-pencil test, the subject has to substitute as many symbols as possible by randomly assigned numbers within 90 seconds. All subjects scored within the normal range for healthy adults, or even higher (normative scores reported in Sheridan et al., 2006: mean 53.2, SD 8.9 for 30-55 year old adults; mean 35.8 SD 9.6 for adults older than 55 years).

\subsection{Audiometric tests}

Both patients and control participants were asked questions about their hearing abilities to ensure that all patients suffered from congenital hearing loss on the right side without any subjective improvement over time and to gain knowledge about the etiology if there was no clear cause reported in their medical records (see Table 2). All patients fulfilled the inclusion criteria of this study by reporting that they had hearing loss in the right ear since birth, although in most patients this was formally tested in early childhood rather than shortly after birth. An otorhinolaryngologist then inspected the tympanic membranes, removed earwax if needed and provided three audiometric tests. First, an objective tympanometry test $(226 \mathrm{~Hz})$ was carried out 
in order to assess the admittance of the tympanic membranes. Next, pure tone audiometry per ear (octave frequencies from 250 to $8000 \mathrm{~Hz}$ in a soundproof room using headphones, masking noise was provided in case of threshold asymmetry $\geq 15 \mathrm{~dB}$ HL) was performed to evaluate hearing. Finally, the presence of otoacoustic emissions (OAEs, objective test of the outer hair cell function; distortion product $\mathrm{OAE}, 1000-8000 \mathrm{~Hz}$ with stimulus $65 / 55 \mathrm{~dB}$ ) was determined.

TABLE 2. Patients and control participants were asked the following questions about their hearing abilities and causes of hearing loss.

\begin{tabular}{|c|l|}
\hline 1. & On which side do you suffer from hearing loss? \\
\hline 2. & When was the onset of this hearing loss according to you and your family? \\
\hline 3. & When was the onset of this hearing loss determined for the first time? \\
\hline 4. & If you close the left ear, can you hear anything with your right ear? \\
\hline 5. & Do you have the impression that the hearing loss has gradually increased? \\
\hline 6. & Has the cause of your hearing loss been determined? If yes, please explain. \\
\hline 7. & $\begin{array}{l}\text { Did you ever receive treatment for this hearing loss (e.g. hearing aid)? If yes, which } \\
\text { one and when? }\end{array}$ \\
\hline
\end{tabular}

Otoscopy was normal in all participants, both patients and controls, as well as tympanic membrane admittance (tympanometry). As shown in Table 1, profound hearing loss in the right ear was confirmed by pure tone audiometry in all patients, whereas they had normal hearing thresholds in the left ear. All control participants had normal hearing in both ears. These results were confirmed by the objective OAE testing: OAEs were absent in the right ear of all patients and present in their left ear, whereas OAEs were present in both ears of control participants.

\section{3 fMRI tasks}

\subsubsection{Task design and procedure}

Word generation task. A silent word generation task was used to assess the lateralization of production. In this widely used paradigm (e.g. Van der Haegen et al., 2011), a letter (b, d, k, 1, m, $\mathrm{n}, \mathrm{p}, \mathrm{r}, \mathrm{s}$ or $\mathrm{t}$ ) is presented in the middle of the screen for 15s. Subjects are asked to mentally generate as many words as possible starting with this letter. In ten control blocks, the Dutch nonword 'baba' had to be silently repeated for 15s. Experimental and control blocks were alternated by $15 \mathrm{~s}$ resting blocks, indicated on the screen by a horizontal line. Prior to scanning, subjects were familiarized with the paradigm by letting them produce words out loud. The mean 
number of words generated per letter was comparable for patients and control subjects group (Patients: Mean per letter $=7.8, \mathrm{SD}=2.4$; Controls: Mean per letter $=7.0, \mathrm{SD}=2.6$ ).

Reading task. The lateralization of reading was measured by a passive viewing task that consisted of three types of stimuli: Words, consonant strings and chequerboards. Subjects were asked to carefully pay attention to all stimuli. In other words, they had to read the words or watch the chequerboards but no response was required. The paradigm was similar to the one used in Cohen et al. (2002). By using minimal task requirements and short presentation durations, the neural signal is presumably mainly evoked by bottom-up visual reading processes instead of emphasizing top-down phonological influences as in for example naming tasks (Dehaene \& Cohen, 2011). There were three sequences of twelve blocks each, with 20 trials per block. Trials within a block were randomized for each subject. The order of blocks within the sequences was also randomized, but identical for all subjects. A 7s resting period separated the three sequences. Each trial started with a fixation cross for $550 \mathrm{~ms}$, immediately followed by a stimulus for 200 ms.

Auditory task. The paradigm to determine the lateralization of auditory perception of verbal and nonverbal information was adopted from Thierry, Giraud and Price (2003). There were four conditions: (1) Verbal sequences with spoken words telling a story (e.g. 'heavy snoring ... mosquito coming by ... person waking ... a switch clicks on ... spraying twice'), (2) noise sequences based on the verbal sequences, (3) nonverbal sequences with environmental sounds telling the same story as in their verbal counterpart, (4) noise sequences based on the nonverbal sequences. All recordings were 15s long. Subjects were asked to indicate whether the story involved an animal or not (which was the case in $50 \%$ of the trials) by pressing a button with their right index or middle finger respectively at the end of the trial when a beep tone was played. In the noise conditions, they also had to press the middle finger button at the end of the trial. The verbal sequences were translated into Dutch and recorded by a male native Dutch speaker. For the nonverbal condition, the original files from Thierry et al. (2003) were used. Noise sequences were created by making the stimuli unintelligible with a signal correlated noise (SCN) script provided by Davis and Johnsrude (2003). SCN is an optimal baseline for auditory speech processing, because it removes linguistic properties but the amplitude envelope and 
spectral profile are the same as in the original sequence (Stoppelman, Harpaz \& Ben-Shachar, 2013). There were two sequences of four blocks (one for each condition). The order was randomized, but identical for all subjects. Each block was followed by a 12.5 s resting period. Subjects heard one example stimulus from each condition before scanning.

All patients and control subjects performed the three fMRI tasks in a fixed order (i.e. word generation, reading and finally the auditory task).

\subsubsection{Image acquisition}

Images were acquired on a 3-Tesla Siemens Trio MRI scanner (Siemens Medical Systems, Erlangen, Germany) with a 32-channel radiofrequency head coil. A high-resolution anatomical image was collected using a T1-weighted 3D MPRAGE sequence (TR = $2250 \mathrm{ms,}$ $\mathrm{TE}=4.18 \mathrm{~ms}$, image matrix $=256 \times 256$, flip angle $=9^{\circ}$, voxel size $=1 \times 1 \times 1 \mathrm{~mm}^{3}$ ) Functional images were then obtained using a T2*-weighted gradient-echo EPI sequence. Forty axial slices covering the whole-brain were acquired $(\mathrm{TR}=2000 \mathrm{~ms}, \mathrm{TE}=28 \mathrm{~ms}$, image matrix $=$ $64 * 64$, flip angle $=80^{\circ}$, slice thickness $=3.0 \mathrm{~mm}$, distance factor $=17 \%$, voxel size $=3.5 * 3.5$ $* 3 \mathrm{~mm}^{3}$, acquired in interleaved even order)

\subsubsection{Data analysis}

Data analysis was performed using SPM8 software (Wellcome Trust Centre for Neuroimaging, London, UK). The first four acquired images of all runs were removed in order to reach a stable magnetic field. Images were first manually reoriented by setting the origin to the anterior commissure (AC). Data preprocessing consisted of (1) slice time correction because slices were acquired in an interleaved way; (2) realignment using rigid body transformations to correct for movement artifacts; (3) coregistration of the anatomical image to the mean functional image; (4) segmentation of the anatomical images into white matter, gray matter and cerebrospinal fluid; (5) normalization of the anatomical (voxel size [1 111$]$ ) and functional images (voxel size [ $\left[\begin{array}{lll}3 & 3 & 3\end{array}\right]$ ) by applying the parameters from the segmentation with a $4^{\text {th }}$ degree 
B-spline interpolation; (6) spatial smoothing with an isotropic Gaussian Kernel of $5 \mathrm{~mm}$ full width at half-maximum.

In first-level analyses, data were convolved with a canonical haemodynamic response function to obtain the BOLD signal, added with the time derivatives. Head movement parameters were added as regressors of no interest. If head movements exceeded the voxel size of $3 \mathrm{~mm}$, the ArtRepair toolbox (Mazaika, Hoeft, Glover \& Reiss, 2009) was used to correct the infected volumes. This was only the case for one subject in the reading and auditory perception task (movements up to $5 \mathrm{~mm}$ ). Statistical parametric maps were calculated by contrasting the most interesting conditions per task using the general linear model (GLM). These were word generation for a target letter vs. repetition of baba for production, passive word reading vs. chequerboard viewing for reading, word vs. word noise and sound vs. sound noise in addition to the meaningful (word and sound combined) vs. noise conditions (word noise and sound noise combined) for auditory perception. Individual lateralization indices (LIs) were calculated for each word condition and its control condition. We used the LI Toolbox 1.02 by Wilke and Lidzba (2007). This toolbox calculates a weighted mean LI in which higher thresholds get a higher weight for the LI, thus avoiding dependence on a certain statistical threshold as in classical LIs based on a normalized difference of number of activated voxels in each hemisphere (see Wilke \& Lidzba, 2007 and Seghier, 2008 for a more detailed description of the methodological advantages of this approach). For each region of interest (ROI), 20 equally sized steps from 0 to the maximum $t$-value were taken. Then, 100 bootstrap resamples with a resample ratio of $k=0.25$ were taken in the left and right investigated area for each level. From the resulting 10.000 possible LI combinations only the central $50 \%$ of data were kept in order to exclude statistical outliers. Finally, a weighted mean LI for each individual was calculated (see Wilke \& Lidzba, 2007). In previous studies (e.g. Van der Haegen, Cai \& Brysbaert, 2012), -0.50 and +0.50 were taken as a cut-off score to consider an LI as clearly RH and LH dominant respectively. For the word generation task, a ROI was created with the Automated Anatomical Labeling (AAL) template (Tzourio-Mazoyer et al., 2002), including the pars opercularis and pars triangularis in the IFG as the combination of these areas is classically seen as Broca's area. For the passive reading task, we used the vOT ROI from Twomey et al. (2011). This ROI ranged from $X=-30$ to $-54, Y=-45$ to -70 and $Z=-30$ to -4 with the exclusion of cerebellar regions 
and restriction to the fusiform and inferior temporal gyri. A mirror-reversed region was added for the RH. For the auditory perception tasks, we created a ROI covering the temporal lobes important for higher-order auditory processing. This ROI included the following LH and RH regions in the AAL template: inferior, middle and superior temporal gyrus, middle and superior temporal pole (see Figure 1 for an illustration of all ROI masks). Subjects showing no activation at an uncorrected $p<0.005$ level in a ROI were excluded because their activity would be too weak to calculate reliable LIs. This was the case for one control subject in the word generation task and one patient in the reading task (his control subject was excluded from the second level analyses as well).

$\mathbf{A}$
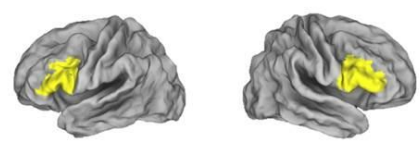

B
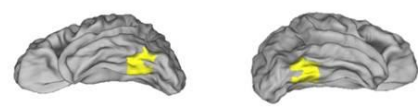

C
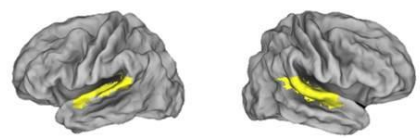

Figure 1. Regions of interest (ROIs) used for the calculation of individual lateralization indices. A: Broca's area as ROI in the word generation task; B: ventral occipito-temporal region as ROI in the reading task; C: temporal lobe regions used as ROI in the auditory perception task.

Random-effect analyses were then performed at group level to compare activity in the abovementioned contrasts between the patients and control subjects. For all contrasts, an uncorrected $p<0.005$ and cluster-level family-wise error-rate (FWE) correction of $p<0.05$ threshold was chosen. For word generation, a two-sampled t-test was performed for the word vs. baba condition. For reading, a two-sample t-test compared the two groups for the word vs. chequerboard contrast. For auditory perception, two-sample t-tests compared the word vs. word noise and sound vs. sound noise conditions. In addition, conjunction analyses at the random 
effects level were performed for all these contrasts in order to reveal common activity in the control subjects and patient group. In these analyses, the contrasts between the relevant conditions from each task mentioned above were simultaneously tested for both groups. Finally, auditory perception ANOVA analyses tested main and interaction effects between group and meaningful vs. meaningless conditions (i.e. word plus sound vs. word noise plus sound noise) and between group and type of auditory stimulus corrected for noise (i.e. word minus word noise vs. sound minus sound noise).

\section{Results}

\subsection{Word generation}

\subsubsection{Group results}

Overall, generating words starting with a target letter compared to the repetition of the non-word baba lead to strong activity in the IFG in the pars opercularis (Brodmann Area [BA] 44) and pars triangularis (BA45) as expected. Activity further extended to the LH putamen, caudate, precentral gyrus (BA9), superior (BA7) and inferior (BA40) parietal lobule and inferior occipital gyrus (BA37), and the anterior (culmen, Lobule IV/V) and posterior (declive, lobule VI) RH cerebellum (all $T>3.05, k$-values $>62$ ). One control subject was excluded from the group analyses, because he did not show any activity in the IFG. Figure 2 shows the activity for the right-sided deaf patients (Figure $2 \mathrm{~A}$, all $T>3.70$, all $k$-values $>120$ ) and control subjects (Figure 2B, all $T>4.03$, all $k$-values $>51$ ) separately. The conjunction analysis showed common activity in the patient and control group in the pars opercularis (BA44) and pars triangularis (BA45) and further in the LH precentral gyrus (BA9), LH supplementary motor area (BA6 and 32), RH globus pallidus and RH cerebellum (anterior part, culmen, Lobule IV/V and posterior part, declive, lobule VI)), and finally bilateral putamen, caudate, and insula (BA13) (all $T>3.11$, $k$-values $>75)$. The two-sampled t-test for patients vs. control subjects in the wordgen $>$ baba contrast did not show any significant differences at our statistical threshold $(p<.005$ whole-brain uncorrected, $p<.05$ FWE cluster-corrected) for patients over controls. Control subjects did show more activity in the left posterior insula (BA13), bilateral precentral gyrus (BA6), right cingulate 
region (BA24), medial occipitotemporal region (BA37), and right superior occipital gyrus (BA19; all $T>3.11$, all $k$-values $>59$ ).

A

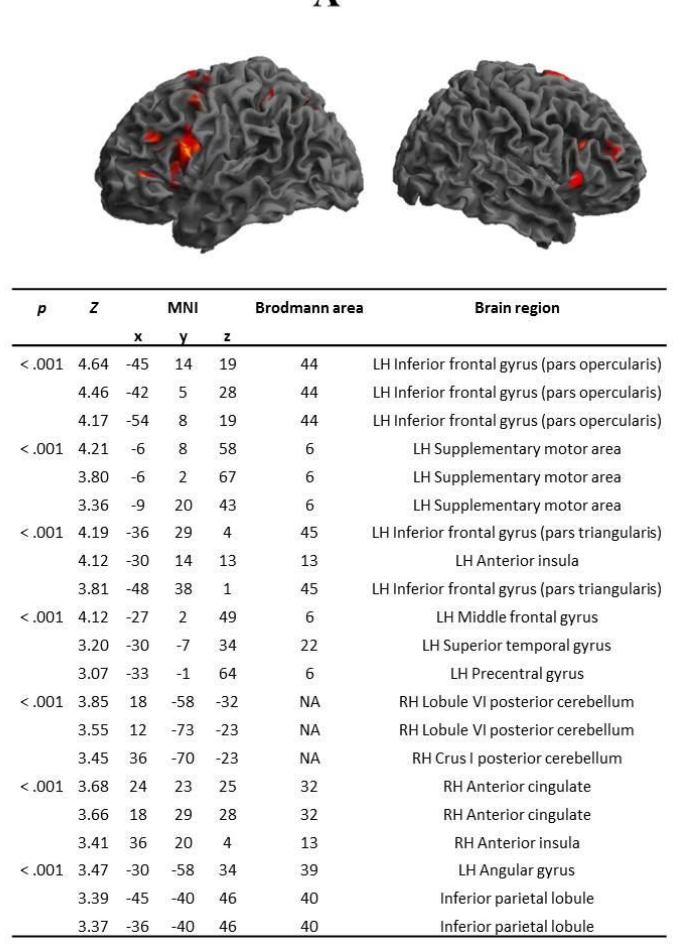

B
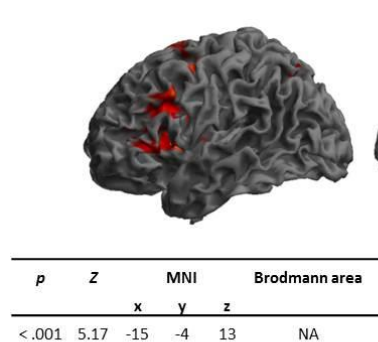

$\begin{array}{cccccc}5.07 & -18 & 8 & 13 & \text { NA }\end{array}$ $\begin{array}{cccc}4.87 & -12 & 14 & 1\end{array}$ $\begin{array}{llll}4.23 & -39 & 26 & 19\end{array}$ $<.001 \quad 4.66 \quad-6 \quad 17 \quad 46$ $\begin{array}{lllll}4.33 & 0 & 14 & 55\end{array}$ $\begin{array}{llll}4.26 & -12 & 14 & 40\end{array}$ $\begin{array}{lllll}.001 & 4.60 & 45 & -61 & -29\end{array}$ $\begin{array}{llll}4.50 & 24 & -73 & -26\end{array}$ $\begin{array}{lllll}3.86 & 36 & -64 & -29\end{array}$ $\begin{array}{lllll}0 & 3.65 & 15 & -43 & -23\end{array}$ $\begin{array}{llll}3.63 & 0 & -58 & -35\end{array}$ $\begin{array}{llll}3.41 & 6 & -34 & -20\end{array}$ $\begin{array}{lllll}<.01 & 3.47 & -27 & -64 & 40\end{array}$ $\begin{array}{llll}3.44 & -24 & -55 & 37\end{array}$ \begin{tabular}{llll}
3.14 & -30 & -67 & 52 \\
\hline
\end{tabular}

(3)
C
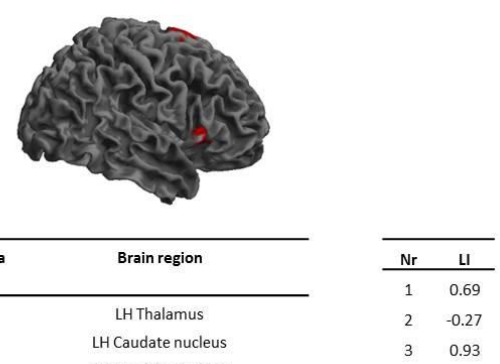

$2 \quad-0.27$

$3 \quad 0.93$

$4 \quad 0.95$

$5 \quad 0.66$

$6 \quad 0.86$

$\begin{array}{ll}7 & 0.85\end{array}$

$\begin{array}{ll}8 & 0.57\end{array}$

$9 \quad 0.74$

$10 \quad 0.51$

11 NA

$12 \quad 0.66$

\begin{tabular}{ll}
13 & 0.67 \\
\hline & 0.93 \\
\hline
\end{tabular}

$14 \quad 0.93$

Figure 2. Group results of the word generation task measuring speech production for the 7 patients (A) and 6 control subjects (B) showing significant activity at an $<0.005$ whole-brain uncorrected, $<0.05$ FWE cluster-corrected level (reported $p$-values corresponding to $T>3.7, k>120$ voxels in A, $T>4.03, k>51$ voxels in $\mathrm{B}$ ). The condition in which subjects silently generated words was contrasted against silent repetition of the Dutch non-word baba. For each cluster, Montréal Neurological Institute (MNI) coordinates are given together with the corresponding Brodmann area, the brain region identified by the AAL-template (Tzourio-Mazoyer et al., 2002) and the cerebellum stereotaxic atlas by Schmahmann et al. (1999). C: Lateralization indices (LIs) per subject calculated by the LI toolbox 1.02 (Wilke \& Lidzba, 2007). Subjects 1 to 7 correspond to the right-sided deaf patients, subjects 8 to 14 are the control subjects. The LI of subject 11 was not calculated due to weak activity (see main text).

\subsubsection{Individual results}

Given the small sample size, individual LIs were of most interest for the current study. They provide a detailed comparison between the lateralization of right-sided deaf patients and controls. Moreover, the weighted mean LI calculation ensures reliable LIs without being 
dependent on statistical thresholds. As such, we avoided drawing conclusions based on grouplevel results that could have been affected by a lack of statistical power in our small sample. Figure 2C gives a list of the LIs calculated for the IFG mask. Six out of 7 patients showed a clear typical LH lateralization for production with scores above +0.50 . One patient with an LI of -0.27 could be considered as bilateral with a trend towards atypical RH lateralization. The six included control subjects all were typically LH lateralized as expected. Overall, patients and controls had a similar lateralization pattern for word production. This was confirmed in a non-parametric Mann-Whitney U-test for independent samples $(p=.37)$.

\section{$3.2 \quad$ Reading}

\subsubsection{Group results}

Taking all subjects together, the passive word reading vs. chequerboard viewing elicited activity in the vOT (BA37), in addition to clusters in the left putamen, left superior motor area (BA6) and left precentral gyrus (BA6; all $T>3.11$, all $k$-values $>122$ ). Figure $3 A$ shows the results for six patients (one patient was excluded because of too weak activity in the vOT, see criteria above, all other $T>4.00, k$-values $>42$ ); his control subject was also excluded from the group level analyses, see Figure 3B, all $T>4.03, k$-values $>44$ ). Note that the vOT region cannot be clearly seen at the group level, presumably because the peaks in the small reading area do not overlap sufficiently in this small group. As a result, the conjunction analysis did not show any significant common activity at our $p<0.005$ whole-brain uncorrected, $p<0.05$ FWE cluster-corrected level. The vOT only reached FWE corrected $p=.37$ in the LH at MNI $=[-39$, $58,-20]$. We therefore focus on the reliably calculated LIs at the individual level (see below). Patients did not show significantly more activity in any brain region compared to controls. The ttest of control subjects' activity over patients only reached significance in the middle occipital region (BA17, $T>3.17, k=68)$. 
A
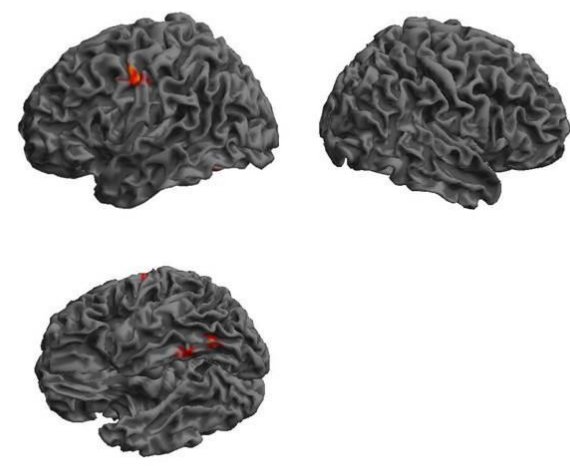

\begin{tabular}{ccccccc}
\hline \multicolumn{1}{c}{$p$} & $\boldsymbol{z}$ & $\mathbf{M N I}$ & \multicolumn{1}{c}{ Brodmann area } & Brain region \\
& & $\mathbf{x}$ & $\mathbf{y}$ & $\boldsymbol{z}$ & & \\
\hline$<0.01$ & 4.39 & -51 & -13 & 40 & 6 & LH Precentral gyrus \\
& 3.98 & -51 & -4 & 46 & 6 & LH Precentral gyrus \\
$<0.01$ & 3.88 & -24 & 2 & 13 & NA & LH Putamen \\
& 3.32 & -27 & 8 & 1 & NA & LH Putamen \\
& 3.23 & -21 & -7 & 19 & NA & LH Putamen \\
0.03 & 3.76 & -39 & -61 & -14 & 37 & LH Ventral occipitotemporal region \\
& 3.47 & -39 & -55 & -23 & 37 & LH Ventral occipitotemporal region \\
& 3.27 & -33 & -43 & -23 & 20 & LH Ventral occipitotemporal region \\
\hline
\end{tabular}

B

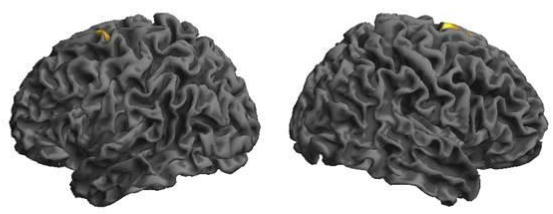

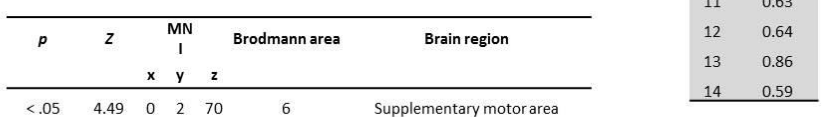

Figure 3. Group results of the passive reading task measuring reading for 6 patients (A) and their 6 corresponding control subjects (B) showing significant activity at an $<0.005$ whole-brain uncorrected, $<0.05$ FWE cluster-corrected level (reported $p$-values corresponding to $T>4.0, k>42$ voxels in A, $T>4.03, k>44$ voxels in B). The contrast shown compares activity during word presentation with activity during passive chequerboard viewing. For the LH, an inferior view of the brain was added to make the ventral occipitotemporal (vOT) region visible. Note that the group results of the control subjects (B) do not clearly show the vOT region, presumably because it is a small region whose exact location can easily differ across subjects. All individual subjects (except patient 1) however showed significant activity in the reading area. We therefore focus on the individual lateralization indices (C, see main text).

\subsubsection{Individual results}

The individual LIs based on activity in the left and right vOT reading regions were again comparable for right-sided deaf patients and control subjects (Figure 3C). Everyone had a clear LH lateralization with values above +0.50 . Only one patient had a slightly weaker LI of +0.38 but still activated his LH most during passive word reading. The LI of one patient was not calculated because of too weak activity in the vOT; the LI of his control subject was included in the individual analysis only. The same distribution of LI values was again confirmed by a MannWhitney U-test $(p=.37)$. 


\subsection{Auditory perception}

\subsubsection{Group results}

First, data were explored for meaningful against meaningless conditions by an ANOVA contrasting activity during listening to sentences and sounds against the noise versions of the same words and sounds. General results for patients and controls together showed activity in the bilateral superior temporal gyrus (BA22), right superior temporal pole (BA22), left middle temporal gyrus (BA21), right anterior cingulate gyrus (BA32), left supplementary motor area (BA6), right IFG (BA44) and precentral gyrus (BA6), left middle occipital gyrus (BA18) and left inferior parietal lobule (BA40; all $T>3.01, k$-values $>98)$. There was significantly more activity observed for patients over controls in the right posterior insula near the claustrum (MNI [39 -13 10], $T=3.05, k=65)$ but nowhere for controls over patients at the $p<0.005$ uncorrected, $p<$ 0.05 FWE cluster-corrected level. The activity pattern illustrates that all subjects activated areas that are usually related to meaningful, higher-order auditory processing. For the ANOVA that tested words controlled for noise against sounds controlled for noise, there was a main effect of patients over controls in the left postcentral gyrus (BA43) and right precentral gyrus (BA6; all $T$ $>2.80, k$-values $>64)$ but again no main effect for controls over patients. Words elicited more activity than sounds (both controlled for noise) in the left middle temporal lobe (BA21) and right superior temporal region (BA22; all $T>2.80, k$-values $>72$ ); sounds compared to words also revealed significant activity in the right superior temporal gyrus (BA22; $T=2.80, k=139$ ). Finally, we report the results for the most specific two-sample t-test contrasts that are also used for the individual LI calculations and the conjunction analyses. Sentence listening compared to the noise control generally resulted in activity in bilateral superior (BA22) and middle temporal gyri (BA21), bilateral superior (BA8) and medial (BA6) frontal gyri, left precentral gyrus (BA6), left insula (BA13), left anterior cerebellum (culmen, Lobule IV/V), left precuneus (BA19) and superior parietal lobule (BA7), as well as in the right anterior cingulate gyrus (BA32), putamen, caudate and globus pallidus (all $T>3.01, k$-values $>67$ ). Patients had more activity in the postcentral gyrus (BA5, $T=3.05, k=77$ ), whereas control subjects had nowhere more activity than patients. When we directly compared the two groups for the sentence listening against noise contrast in the conjunction analysis at the uncorrected $p<0.005$ whole-brain level, $p<0.05$ 
FWE cluster-corrected level, there was significant activity $(T \mathrm{~s}=3.05, k=183)$ in the $\mathrm{LH}$ and RH superior and middle temporal gyri (BA21 and 22) as expected. Sound listening compared to the noise control gave activity in bilateral superior (BA41 in LH and BA42 in RH) and middle temporal gyri (BA21), bilateral insula (BA13), bilateral occipital cuneus (BA17-18), LH thalamus and putamen, and RH inferior (BA47) and middle frontal gyrus (BA9; all $T>3.01, k$ values > 82). No main effects of group were observed in the two-sample t-test. The conjunction analysis looking for common activity in the patient and control group revealed significant activity $(T=3.05, k=106)$ in the $\mathrm{LH}$ and $\mathrm{RH}$ superior and middle temporal gyri (BA41 and 22), similar to the sentence listening against noise contrast. Figure 4 displays the group activity for patients (4A, all $T>3.01, k$-values $>67)$ and control subjects (4B, all $T>3.71, k$-values $>57)$ for the word against noise condition. Figure 5 shows the group results for the sound against noise condition (patients in 5A, all $T>3.71, k$-values $>43$; control subjects in 5B, all $T>3.71, k$ values > 222).

A
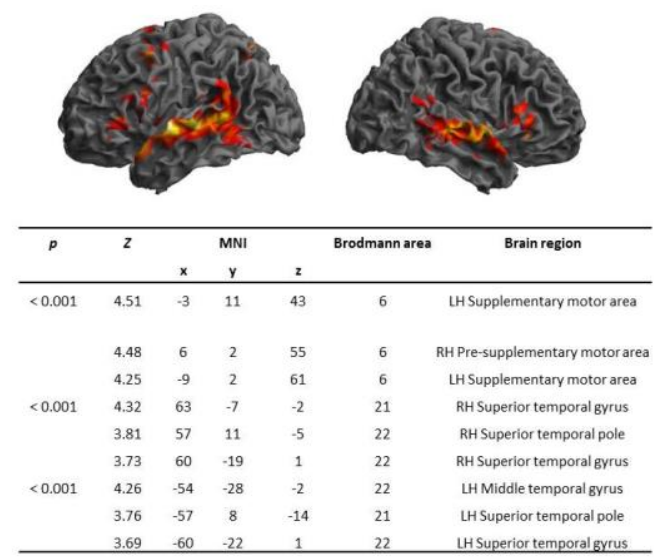

B

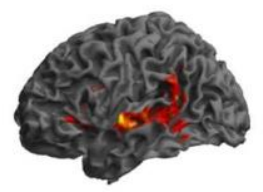

\begin{tabular}{|c|c|c|c|c|c|c|}
\hline \multirow[t]{2}{*}{ p } & \multirow[t]{2}{*}{$z$} & \multicolumn{3}{|c|}{ MNI } & \multirow{2}{*}{ Brodmann area } & \multirow[t]{2}{*}{ Brain region } \\
\hline & & $x$ & $\mathrm{y}$ & 2 & & \\
\hline \multirow[t]{3}{*}{$<.001$} & 4.38 & 57 & -40 & 1 & 22 & RH Middle temporal gyrus \\
\hline & 4.28 & 66 & -19 & 1 & 22 & RH Superior temporal gyrus \\
\hline & 4.22 & 54 & 14 & -5 & 22 & RH Superior temporal gyrus \\
\hline \multirow[t]{3}{*}{$<.001$} & 4.30 & -51 & 17 & 13 & 44 & LH Inferior frontal gyrus (pars opercularis) \\
\hline & 4.03 & -39 & 2 & 31 & 6 & LH Precentral gyrus \\
\hline & 3.54 & -45 & 14 & -2 & 47 & LH Inferior frontal gyrus (pars orbitalis) \\
\hline \multirow[t]{3}{*}{$<.001$} & 4.29 & -57 & -34 & 1 & 22 & LH Middle temporal gyrus \\
\hline & 4.18 & -57 & -16 & 1 & 22 & LH Superior temporal gyrus \\
\hline & 4.14 & -54 & -19 & -8 & 21 & LH Middle temporal gyrus \\
\hline \multirow[t]{3}{*}{$<.05$} & 3.71 & 21 & 8 & 10 & NA & RH Putamen \\
\hline & 3.54 & 15 & 14 & 4 & NA & RH Caudate nucleus \\
\hline & 3.44 & 12 & 5 & 10 & NA & RH Caudate nucleus \\
\hline \multirow[t]{3}{*}{$<.01$} & 3.61 & 30 & -70 & 40 & 19 & RH Precuneus \\
\hline & 3.45 & 39 & -52 & 40 & 40 & RH inferior parietal lobule \\
\hline & 3.41 & 42 & -61 & 43 & 40 & RH Inferior parietal lobule \\
\hline \multirow[t]{3}{*}{$<.01$} & 3.47 & -15 & 14 & 7 & NA & LH Caudate nucleus \\
\hline & 3.42 & -18 & -1 & 10 & NA & LH Globus pallidus \\
\hline & 3.41 & -12 & 8 & 1 & NA & LHCaudate nucleus \\
\hline
\end{tabular}

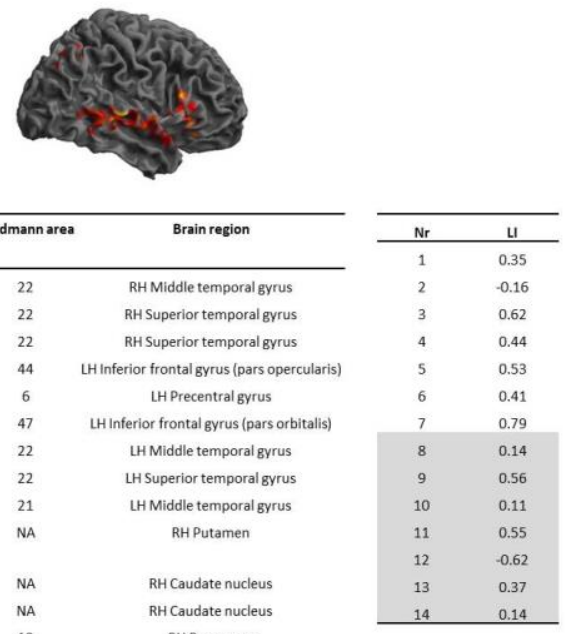

C 
A
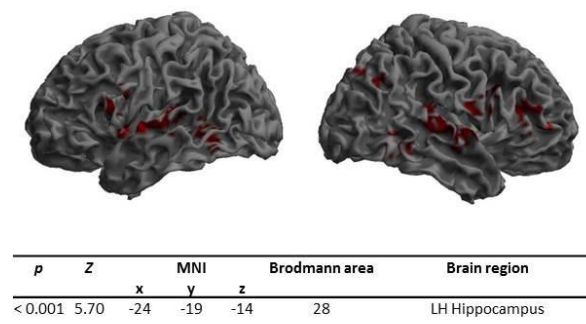

$\begin{array}{llll}4.35 & -30 & -4 & -17\end{array}$

$\begin{array}{lllll}4.23 & -39 & 2 & -2\end{array}$ $\begin{array}{lllll}0.001 & 4.73 & 63 & -7 & -2\end{array}$

$\begin{array}{lllll}4.10 & 60 & -25 & 13 & 42\end{array}$

$\begin{array}{llll}4.04 & 27 & -16 & 13\end{array}$

$\begin{array}{llllll}<0.001 & 4.41 & -51 & -19 & 7 & 41\end{array}$

$\begin{array}{lllll}4.40 & -63 & -7 & 1 & 22\end{array}$

$\begin{array}{lllll}4.06 & -63 & -28 & 1 & 21\end{array}$

$\begin{array}{llllll}<0.01 & 4.28 & 45 & 53 & 4 & 10\end{array}$

$\begin{array}{lllll}3.57 & 51 & 44 & -2 & 45\end{array}$

$\begin{array}{lllll}3.05 & 42 & 32 & 1 & 45\end{array}$

$\begin{array}{llllll}<0.01 & 4.00 & -60 & 2 & 19 & 6\end{array}$

$\begin{array}{lllll}3.64 & -54 & 11 & 10 & 6\end{array}$

$\begin{array}{lllll}3.54 & -51 & -1 & 25 & 9\end{array}$

$\begin{array}{lllllll}<0.01 & 3.95 & 48 & 8 & 28 & 44\end{array}$

$\begin{array}{lllll}3.70 & 54 & 14 & 40 & 6\end{array}$

$\begin{array}{lllll}3.51 & 39 & 5 & 28 & 9\end{array}$

$\begin{array}{llllll}<0.01 & 3.93 & 30 & -76 & 40 & 19\end{array}$

$\begin{array}{lllll}3.71 & 36 & -64 & 31 & 39\end{array}$

$\begin{array}{lllll}3.05 & 48 & -61 & 37 & 40\end{array}$

$<\begin{array}{llllll}0.001 & 3.85 & 60 & -52 & -2 & 37\end{array}$

$\begin{array}{lllll}3.74 & 45 & -34 & -14 & 20\end{array}$

$\begin{array}{lllll}3.62 & 51 & -46 & -14 & 37\end{array}$

$\begin{array}{llllll}<0.001 & 3.84 & 48 & 38 & 16 & 45\end{array}$

$\begin{array}{lllll}3.82 & 57 & 32 & 7 & 45\end{array}$

$\begin{array}{lllll}3.78 & 39 & 32 & 10 & 45\end{array}$

$\begin{array}{lllllll}0.05 & 3.82 & 57 & 14 & 13 & 44\end{array}$

$\begin{array}{llllll}3.80 & 57 & -1 & 16 & 6\end{array}$

$\begin{array}{lllll}3.05 & 54 & 17 & 22 & 45\end{array}$

$\begin{array}{lllll}<0.05 & 3.15 & 6 & -4 & 64\end{array}$

$\begin{array}{llll}3.14 & -6 & 14 & 55\end{array}$

$\begin{array}{lllll}3.10 & 6 & 2 & 58\end{array}$

RH Putamen

LHSuperior temporal gyrus

LHSuperior temporal gyrus

LH Middle temporal gyrus

RH MIddle frontal gyrus

RH Inferior frontal gyrus

RH Inferior frontal gyrus

LH Precentral gyrus

LH Precentral gyrus

LH Middle frontal gyrus

RH Inferior frontal gyrus

RH Middle frontal gyrus

RH Middle frontal gyrus

RH Precuneus

RH Inferior parietal Lobule

RH Middle temporal gyrus

RH Fusiform gyrus

RH Inferior temporal lobe

RH Inferior frontal gyrus

RH Inferior frontal gyrus

RH Inferior frontal gyrus

RH Inferior frontal gyrus

RH Precentral gyrus

RH Inferior frontal gyrus

RH Supplementary motor area

LH Supplementary motor area
B

C
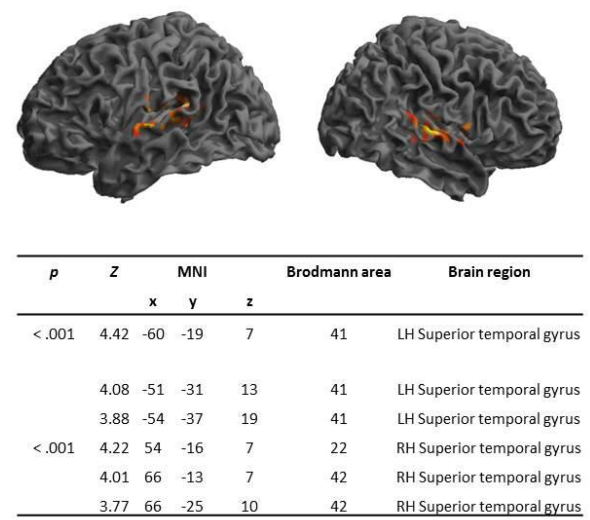

RH Middle temporal gyrus

RH Supplementary motor area

Figure 5. Group results of the auditory processing task for 7 patients (A) and their 7 matched control subjects (B) showing significant activity during the sound listening versus noise condition at an $<0.005$ whole-brain uncorrected, $<0.05$ FWE cluster-corrected level (reported $p$-values corresponding to $T>3.71, k>43$ voxels in A, $T>3.71, k>$ 222 voxels in B). C: Lateralization indices (LIs) per subject. 


\subsubsection{Individual results}

Individual LIs were calculated with the broad temporal ROI covering areas associated with higher-order, semantically related auditory processing. We expected more LH activity for word processing and more $\mathrm{RH}$ activation for sound processing. As can be seen in Figures $4 \mathrm{C}$ and $5 \mathrm{C}$, the results are more mixed than for the production and reading tasks. For words, three out of seven patients were clearly LH lateralized with LI values above +0.50 , another three were also LH lateralized with LIs between 0.35 and 0.44 and the last patient had a more bilateral LI towards the RH with a score of -0.16 . However, our paradigm elicited a similar pattern in the control subjects: two out of seven subjects had LH dominant LIs above +0.50 , four of them had LIs between 0.11 and 0.37 and one was $\mathrm{RH}$ dominant with an LI of -0.62 . LI values were comparable across the patient and control subject group (Mann-Whitney U-test: $p=.26$ ). Sounds were indeed more lateralized to the other hemisphere, even though we again found a mixed pattern. In patients, none of the LIs pointed to a clear RH dominance, three out of seven can be classified as bilateral with a trend towards the $\mathrm{RH}$, three showed a trend towards the $\mathrm{LH}$ and the remaining one was clearly LH dominant. In control subjects, only one LI below -0.50 was observed, four out of seven were bilateral with an $\mathrm{RH}$ dominant trend and two with an LH dominant trend. Most importantly for the current research question, the pattern was again comparable across groups (Mann-Whitney U-test: $p=.17$ ).

\section{Discussion}

Patients who are deaf on one side from birth can provide unique information about the organization of lateralized functions. Based on a single patient, Adorni et al. (2013) ventured that congenital absence of auditory input from the right ear may result in atypical, right hemisphere language dominance. Similarly, Burton et al. (2013) presented evidence that congenital absence of auditory input may lead to an excessive stimulation of the contralateral auditory pathway of the hearing ear. To test the possibility properly, we tested 7 participants with congenital right ear deafness on three established fMRI language paradigms - word generation, sentence perception, and word reading - that are known to show clear left hemisphere asymmetries. These tasks allowed us to address to what extent the laterality of auditory language perception is different in people without input from the right ear. They also allowed us to examine the colateralization of 
the various functions. In particular, we were interested to see whether the lateralization of Broca's area would be different in right ear deaf patients as well, as the genetic influences on laterality are usually formulated with respect to speech production. So, theoretically it was quite possible that speech perception (and reading) were right lateralized in patients with right ear deafness, but that speech production nevertheless was left dominant.

As it happened, we failed to find any evidence corroborating Adorni et al.'s (2013) conjecture. There was some evidence for atypical dominance in one patient (patient 2), but this probability was not larger than that observed in the control participants and also not larger than expected on the basis of atypical hemisphere dominance in the right-handed population. If each right-hander has an a priori chance of $5 \%$ of atypical language dominance, chances of observing at least one patient with atypical language dominance in a group of 7 is $30 \%$ (with $26 \%$ chance of observing exactly one patient with atypical dominance). Interestingly, even for auditory speech perception, there was little evidence that exclusive input from the left ear made much difference to the laterality of the regions involved.

We had the highest chances of finding a difference between the patients and the control group in the auditory task. In this task, participants listened to speech and environmental sounds with brain activity controlled by signal correlated noise. All subjects activated the expected regions in the temporal lobes. If semantic decisions are required, activity is typically widespread from the posterior and middle STG until the anterior part with a LH dominance for speech and RH dominance for sounds. Sentence listening indeed lateralized to the LH even though one patient and one control activated their RH hemisphere more. For sounds, five out of seven patients and three out of seven controls showed a RH dominance, even though the degree of lateralization was rather weak with only two subjects having LIs larger than $+/-0.50$. At the overall group level, the activation locations are comparable to those previously reported in auditory perception studies (e.g. Thierry \& Price, 2006, from whom the paradigm and environmental sound stimuli were adapted). The interpretation of literature on auditory processing is complicated, because the various studies have presented a wide range of stimulus types going from pure tones, vowels or syllables to lexical, semantic and sentence stimuli, which are lateralized to a different extent. This is also true for the prior literature on auditory processing 
in unilateral hearing impaired subjects (see Introduction for studies using different neuroimaging techniques, different stimuli and paradigms). In general, lateralization tends to go more towards the LH from phonetic to lexical-semantic processing (see Specht, 2013 for a review). According to Poeppel's (2003) asymmetric sampling in time hypothesis, this continuum is due to differences in the temporal properties of the acoustic signal, with the LH being more involved in processing fast changing characteristics such as in words and the RH being more sensitive to longer durations and changes in pitch such as in sounds. The exact nature of lateralization in auditory processes is however still far from clear (see e.g. Arsenault \& Buchsbaum, 2015; Scott $\&$ McGettigan, 2013). In this study, we chose to assess the hemispheric specialization of higherorder cognitive functions, in line with Adorni et al. (2013).

Most important for the current study are the individual LIs (which are rarely reported in other studies) of the patients and controls. It is clear from Figures $4 \mathrm{C}$ and $5 \mathrm{C}$ that both groups produced a similar pattern. This was confirmed by the conjunction analyses showing that patients and control subjects had common activity in the regions of interests. Congenital unilateral rightsided deafness hence does not systematically lead to a reorganization of higher-order auditory processing in terms of laterality in the temporal lobes. This goes against Gordon et al.'s (2013) claim that unique input from the left ear would lead to overactivation of the right hemisphere auditory cortex (but note that their study included children with congenital bilateral hearing loss instead of unilateral deaf adults), and against Jensen et al.'s (1989) right ear advantage hypothesis according to which right ear deafness is particularly disadvantageous. Our results are more in line with the observation that the ipsilateral connections of the spared ear become stronger, so that the functioning ear activates both hemispheres (Burton et al., 2012; Hine et al., 2008). Future research will be needed to confirm this hypothesis and test the consequences of a cochlear implant for language laterality in congenital unilateral deaf patients.

Given that there was no effect of right ear deafness on the laterality of speech perception, little effect should be expected on speech production. Indeed, all but one participant had their speech production center in the left IFG, as expected on the basis of previous tests with the paradigm (Van der Haegen et al., 2011). Only patient 2 showed some evidence towards right hemisphere dominance (although a laterality index of -0.27 seems to be more in line with 
bilateral involvement). So, again there is no indication that a lack of auditory input on the right side systematically leads to atypical RH language dominance. As a matter of fact, it seems likely that the left hemisphere dominance for speech perception in the patients is due to the fact that their speech production is left lateralized. Indeed, according to the colateralization account (Cai, Lavidor, Brysbaert, Paulignan, \& Nazir, 2008; Van der Haegen et al., 2012), it is advantageous for the brain to have speech production and speech perception lateralized to the same hemisphere so that interactions between these regions do not require interhemispheric transfer.

It is not surprising that all participants activated their vOT more in the LH than the RH during reading, given that speech production and speech perception were also left lateralized. For reading, the left hemisphere is additionally advantaged because more information is picked up from the visual field right of fixation than left of fixation (Cai et al., 2008). The reading results contradict the study by Adorni et al. (2013) who found an LI of -0.47 for the N170 ERP component in a congenital right-ear deaf woman. The pattern could not be replicated in our seven patients tested in fMRI paradigms with more spatially detailed information. It is unclear what exactly caused the difference in results. It could of course be that the single case was exceptional such as our patient 2. After all, there is a chance of 1 out of 20 that any right-hander has atypical language dominance (Knecht et al., 2000; Loring et al., 1990).

We acknowledge that our study has a few limitations and that the LI patterns are not completely consistent. The hearing performance results (see Table 1) confirmed profound deafness in the right ear and we have every reason to believe this was present from birth, but no tests were done at the time to be $100 \%$ sure. In theory, better informed tests should be possible in the future, now that all babies are tested.

Another limitation is that we could only investigate 7 patients. Given the low prevalence of these patients, this is a unique sample, which allows us to reject the strong conjectures made by Adorni et al. (2013) and Gordon et al. (2013), but which does not allow us to claim that right ear deafness has no effect at all on brain dominance. Maybe right hemisphere dominance changes from $5 \%$ to $10 \%$ ? Or it is more likely in left-handed individuals? Many more data will need to be collected to answer these questions. In particular, individual data would reveal interesting information, but they were unfortunately rarely reported in prior studies. 
With respect to the degree of lateralization, the auditory LIs were weaker than production and reading LIs. As discussed above the exact involvement of $\mathrm{LH}$ and $\mathrm{RH}$ temporal regions in auditory processing still needs a lot of research especially at the individual level. Language subprocesses have a different degree of lateralization (see also Seghier, Kherif, Josse \& Price, 2011) and more bilateral processing for auditory compared to visual reading or production information is definitely plausible, especially because a considerable part of auditory input is transferred via ipsilateral in addition to contralateral pathways. Also note that the currently reported reading LIs are more extreme than those reported for left-handers in our previous study (Van der Haegen et al., 2012). This could be due to the fact that clearer fMRI LIs are obtained in blocked designs (current study) than in event-related designs (previous study), even though in both studies individuals with weak overall activity in the vOT region of interest were excluded. Finally, our group level results revealed that patients recruited their RH insula more when comparing auditory meaningful (words and sounds) to meaningless (noise conditions) stimuli and their LH postcentral and RH precentral gyrus more when contrasting word listening against noise. The insula has been related to syntactic processing in speech comprehension. Thus, it could be that the unilateral deaf patients needed that region in their non-dominant hemisphere more to perform the task. The rolandic cortex (pre- and postcentral cortex) is involved in auditory-motor feedback (Price, 2012). It is not clear why patients recruited that region more, but it could also have to do with the fact that subjects had to respond with a button press to indicate their semantic decision. For production, control subjects showed more activity in regions related to response monitoring (cingulate region), articulatory coding (insula) and motor execution (precentral gyrus) and in visual occipital regions for both production and reading. Again, it is hard to interpret these data in a small sample, but it seems that unilateral deaf patients use a broader network for auditory processing, whereas normal hearing subjects use more areas for production and reading. The most interesting regions of interest did however not show any group effects.

All results discussed above could not be attributed to weaker behavioral performance in our patients. On the contrary, patients were on average slightly better at the control tasks involving word and pseudoword reading and word generation. In general, unilateral hearing loss has been associated with decreased performance on other domains such as hearing in noise, 
sound localization, general academic performance, verbal IQ, self-esteem and exhaustion (Kuppler, Lewis \& Evans, 2013; Lieu, 2013; see Vila \& Lieu, 2015 for a review), even though the patients do not always suffer from their hearing loss in daily life activities. It could be interesting for future research to further explore whether cross-modal tasks involving auditory processing are more affected than isolated language functions. The study of Schmithorst et al. (2014) for example let children with unilateral hearing loss listen to a description of the position of geometric figures while simultaneously showing the configuration on a screen. They found decreased activity in both temporal and occipital regions. It would be interesting to examine such data at an individual level, certainly with participants who show evidence for atypical brain organization. Non-language higher-order processes in unilateral deaf patients are also unexplored. For example, visuo-spatial processing could be affected because auditory attention is constantly directed to the left side and visuo-spatial attention has been found to lateralize to the RH, opposite to the LH speech dominant hemisphere (e.g. Cai, Van der Haegen \& Brysbaert, 2013). In a preliminary behavioral test, we asked our subjects to perform the Schenkenberg bisection task (Schenkenberg, Bradford \& Ajax, 1980), in which they had to draw a vertical line in the middle of 20 horizontal lines. Control subjects misjudged the line center on average by $2.1 \%$ to the right, patients had a rightward bias of $1.41 \%$ but the individual variability was too high to draw reliable conclusions from this small sample (standard deviations of 20 and 18 for controls and patients respectively). Iturria-Medina et al. (2011) argued that the RH houses more general functions that require interconnectivity such as visuo-spatial processing, whereas the LH consists of more centrally organized nodes responsible for specialized functions such as language and motor functions. This may be a framework to assume that RH visuo-spatial attention might be more easily affected by sensory deprivation than the currently tested LH dominant language functions.

The results of Iturria-Medina et al. (2011) were based on diffusion-weighted images. Anatomical data could indeed also reveal other unique information about possible changes in our congenital right-ear deaf patients. Greve et al. (2013) showed that atypical language lateralization in left-handers leads to deviating asymmetries in grey matter in temporal regions and the vOT but not in the IFG despite the fact that the left-handers were classified as being atypically lateralized based on the functional word production paradigm used in this paper. There 
are very few studies investigating white matter connections in adults with unilateral hearing loss. Wu et al. (2009) tested 12 subjects between 8 and 29 years old with either left or right ear deafness and found decreased fractional anisotropy and increased mean diffusivity in the lateral lemniscus and inferior colliculus.

To conclude, our data reveal that congenital right ear deafness does not lead to atypical language lateralization for auditory word and sound perception, word production and word reading. All three functions colateralize in the LH for most patients. Future studies on laterality with these patients may still be interesting to further explore alternative explanations for hemispheric language dominance (e.g. genetic influences may overrule the influence of unilateral sensory deprivation from birth), behavioral consequences of hearing loss and the organization of non-language functions in single-sided deafness. 


\section{Acknowledgements}

We would like to thank Guillaume Thierry (Bangor University) for sharing the auditory nonverbal stimuli and Matt Davis (University of Cambridge) for his advice and SCN script to create the noise conditions in the auditory perception task. The present research was funded by an Odysseus grant awarded by the Government of Flanders to Marc Brysbaert.

\section{References}

Adorni, R., Manfredi, M., \& Proverbio, A. M. (2013). Congenital unilateral deafness affects cerebral organization of reading. Brain sciences, 3(2), 908-922. doi: 10.3390/brainsci3020908

Arsenault, J. S., \& Buchsbaum, B. R. (2015). Distributed neural representations of phonological features during speech perception. The Journal of neuroscience : The official journal of the Society for Neuroscience, 35(2), 634-642. doi: 10.1523/JNEUROSCI.2454-14.2015

Behrmann, M., \& Plaut, D. C. (2015). A vision of graded hemispheric specialization. Annals of the New York Academy of Sciences. Online prepublication DOI: 10.1111/nyas.12833.

Brus, B., \& Voeten, M. (1991). Een-minuut-test vorm A en B, schoolvorderingstest voor de technische leesvaardigheid bestemd voor groep 4 tot en met 8 van het basisonderwijs. Verantwoording en handleiding. Lisse: Swets \& Zeitlinger.

Burton, H., Firszt, J. B., Holden, T., Agato, A., \& Uchanski, R. M. (2012). Activation lateralization in human core, belt, and parabelt auditory fields with unilateral deafness compared to normal hearing. Brain Research, 1454, 3347. doi:

10.1016/j.brainres.2012.02.066

Cai, Q., Lavidor, M., Brysbaert, M., Paulignan, Y., \& Nazir, T. A. (2008). Cerebral Lateralization of Frontal Lobe Language Processes and Lateralization of the Posterior Visual Word Processing System. Journal of Cognitive Neuroscience, 20(4), 672-681.

Cai, Q., Van der Haegen, L., \& Brysbaert, M. (2013). Complementary hemispheric specialization for language production and visuospatial attention. Proceedings of the National Academy of Sciences of the United States of America, 110(4), E322-E330. doi: 10.1073/pnas.1212956110 
Cantlon, J. F., Pinel, P., Dehaene, S., \& Pelphrey, K. A. (2011). Cortical Representations of Symbols, Objects, and Faces Are Pruned Back during Early Childhood. Cerebral Cortex, 21(1), 191-199. doi: 10.1093/cercor/bhq078

Cohen, L., Dehaene, S., Naccache, L., Lehericy, S., Dehaene-Lambertz, G., Henaff, M. A., \& Michel, F. (2000). The visual word form area - Spatial and temporal characterization of an initial stage of reading in normal subjects and posterior split-brain patients. Brain, 123, 291-307. doi: 10.1093/brain/123.2.291

Cohen, L., Lehericy, S., Chochon, F., Lemer, C., Rivaud, S., \& Dehaene, S. (2002). Languagespecific tuning of visual cortex functional properties of the Visual Word Form Area. Brain, 125, 1054-1069. doi: 10.1093/brain/awf094

Davis, M. H., \& Johnsrude, I. S. (2003). Hierarchical processing in spoken language comprehension. Journal of Neuroscience, 23(8), 3423-3431.

Dehaene, S., \& Cohen, L. (2011). The unique role of the visual word form area in reading. Trends in Cognitive Sciences, 15(6), 254-262. doi: 10.1016/j.tics.2011.04.003

Dorothy, V. M. B. (2013). Cerebral asymmetry and language development: cause, correlate, or consequence? Science (New York, N.Y.), 340(6138), 1230531. doi: 10.1126/science. 1230531

Firszt, J. B., Ulmer, J. L., \& Gaggle, W. (2006). Differential representation of speech sounds in the human cerebral hemispheres. The Anatomical Record Part A: Discoveries in Molecular, Cellular, and Evolutionary Biology, 288(4), 345-357. doi: 10.1002/ar.a.20295

Gordon, K. A., Wong, D. D. E., \& Papsin, B. C. (2013). Bilateral input protects the cortex from unilaterally-driven reorganization in children who are deaf. Brain, 136, 1609-1625. doi: 10.1093/brain/awt052

Greve, D. N., Van der Haegen, L., Cai, Q., Stufflebeam, S., Sabuncu, M. R., Fischl, B., \& Brysbaert, M. (2013). A Surface-based Analysis of Language Lateralization and Cortical Asymmetry. Journal of Cognitive Neuroscience, 25(9), 1477-1492. doi:

10.1162/jocn_a_00405

Hervé, P.-Y., Zago, L., Petit, L., Mazoyer, B., \& Tzourio-Mazoyer, N. (2013). Revisiting human hemispheric specialization with neuroimaging. Trends in Cognitive Sciences, 17(2), 6980. doi: 10.1016/j.tics.2012.12.004 
Hine, J., Thornton, R., Davis, A., \& Debener, S. (2008). Does long-term unilateral deafness change auditory evoked potential asymmetries? Clinical Neurophysiology, 119(3), 576586.

Iturria-Medina, Y., Perez Fernandez, A., Morris, D. M., Canales-Rodriguez, E. J., Haroon, H. A., Garcia Penton, L., . . . Melie-Garcia, L. (2011). Brain Hemispheric Structural Efficiency and Interconnectivity Rightward Asymmetry in Human and Nonhuman Primates. Cerebral Cortex, 21(1), 56-67. doi: 10.1093/cercor/bhq058

Jensen, J.H., Johansen, P.A., \& Borre, S. (1989). Unilateral sensorineural hearing loss in and auditory performance with respect to right/left differences. British Journal of Audiology, 23, 215-220. doi:10.3109/03005368909076502

Knecht, S., Drager, B., Deppe, M., Bobe, L., Lohmann, H., Floel, A., . . Henningsen, H. (2000). Handedness and hemispheric language dominance in healthy humans. Brain, 123, 25122518. doi: 10.1093/brain/123.12.2512

Kuppler, K., Lewis, M., \& Evans, A. K. (2013). A review of unilateral hearing loss and academic performance: is it time to reassess traditional dogmata? International journal of pediatric otorhinolaryngology, 77(5), 617-622. doi: 10.1016/j.ijporl.2013.01.014

Leroy, F., Cai, Q., Bogart, S. L., Dubois, J., Coulon, O., Monzalvo, K., . . Dehaene-Lambertz, G. (2015). New human-specific brain landmark: The depth asymmetry of superior temporal sulcus. Proceedings of the National Academy of Sciences, 112(4), 1208-1213. doi: 10.1073/pnas.1412389112

Lieu, J. E. (2013). Unilateral hearing loss in children: speech-language and school performance. B-ENT, Suppl 21, 107-115.

Loring, D. W., Meador, K. J., Lee, G. P., Murro, A. M., Smith, J. R., Flanigin, H. F., . . King, D. W. (1990). Cerebral language lateralization - evidence from intracarotid amobarbital testing. Neuropsychologia, 28(8), 831-838. doi: 10.1016/0028-3932(90)90007-b

Mazaika, P., Hoeft, F., Glover, G.H., \& Reiss, A.L. (2009). Methods and Software for fMRI Analysis for Clinical Subjects . Poster presented at Human Brain Mapping conference 2009.

Oldfield, R. C. (1971). The assessment and analysis of handedness: The Edinburgh Inventory. Neuropsychologia, 9(1), 97-113. doi: 10.1016/0028-3932(71)90067-4 
Pinel, P., Lalanne, C., Bourgeron, T., Fauchereau, F., Poupon, C., Artiges, E., .. . Dehaene, S. (2014). Genetic and Environmental Influences on the Visual Word Form and Fusiform Face Areas. Cerebral Cortex. doi: 10.1093/cercor/bhu048

Poeppel, D. (2003). The analysis of speech in different temporal integration windows: cerebral lateralization as 'asymmetric sampling in time'. Speech Communication, 41(1), 245-255. doi: 10.1016/s0167-6393(02)00107-3

Price, C. J. (2012). A review and synthesis of the first 20 years of PET and fMRI studies of heard speech, spoken language and reading. Neuroimage, 62(2), 816-847. doi:

10.1016/j.neuroimage.2012.04.062

Rodd, J. M., Davis, M. H., \& Johnsrude, I. S. (2005). The neural mechanisms of speech comprehension: fMRI studies of semantic ambiguity. Cerebral Cortex, 15(8), 1261-1269. doi: 10.1093/cercor/bhi009

Schenkenberg, T., Bradford, D. C., \& Ajax, E. T. (1980). Line bisection and unilateral visual neglect in patients with neurologic impairment. Neurology, 30(5), 509-517.

Schmahmann, J. D., Doyon, J., McDonald, D., Holmes, C., Lavoie, K., Hurwitz, A. S., . . . Petrides, M. (1999). Three-dimensional MRI atlas of the human cerebellum in proportional stereotaxic space. Neuroimage, 10(3), 233-260. doi:

10.1006/nimg.1999.0459

Schmithorst, V. J., Plante, E., \& Holland, S. (2014). Unilateral deafness in children affects development of multi-modal modulation and default mode networks. Frontiers in Human Neuroscience, 8, 164. doi: 10.3389/fnhum.2014.00164

Scott, S. K., \& McGettigan, C. (2013). Do temporal processes underlie left hemisphere dominance in speech perception? Brain and Language, 127(1), 36-45. doi: 10.1016/j.bandl.2013.07.006

Seghier, M. L. (2008). Laterality index in functional MRI: methodological issues. Magnetic Resonance Imaging, 26(5), 594-601. doi: 10.1016/j.mri.2007.10.010

Seghier, M. L., Kherif, F., Josse, G., \& Price, C. J. (2011). Regional and Hemispheric Determinants of Language Laterality: Implications for Preoperative fMRI. Human Brain Mapping, 32(10), 1602-1614. doi: 10.1002/hbm.21130 
Seghier, M. L., \& Price, C. J. (2011). Explaining Left Lateralization for Words in the Ventral Occipitotemporal Cortex. Journal of Neuroscience, 31(41), 14745-14753. doi: 10.1523/jneurosci.2238-11.2011

Smith, A. (1973). Symbol Digit Modalities Test manual. Los Angeles, CA, Western Psychological Service.

Specht, K. (2013). Mapping a lateralization gradient within the ventral stream for auditory speech perception. Frontiers in Human Neuroscience, 7, 629. doi: 10.3389/fnhum.2013.00629

Stoppelman, N., Harpaz, T., \& Ben-Shachar, M. (2013). Do not throw out the baby with the bath water: choosing an effective baseline for a functional localizer of speech processing. Brain and behavior, 3(3), 211-222. doi: 10.1002/brb3.129

Thierry, G., Giraud, A. L., \& Price, C. (2003). Hemispheric dissociation in access to the human semantic system. Neuron, 38(3), 499-506. doi: 10.1016/s0896-6273(03)00199-5

Thierry, G., \& Price, C. J. (2006). Dissociating verbal and nonverbal conceptual processing in the human brain. Journal of Cognitive Neuroscience, 18(6), 1018-1028. doi: 10.1162/jocn.2006.18.6.1018

Twomey, T., Duncan, K. J. K., Price, C. J., \& Devlin, J. T. (2011). Top-down modulation of ventral occipito-temporal responses during visual word recognition. Neuroimage, 55(3), 1242-1251. doi: 10.1016/j.neuroimage.2011.01.001

Tzourio-Mazoyer, N., Landeau, B., Papathanassiou, D., Crivello, F., Etard, O., Delcroix, N., .. . Joliot, M. (2002). Automated anatomical labeling of activations in SPM using a macroscopic anatomical parcellation of the MNI MRI single-subject brain. Neuroimage, 15(1), 273-289. doi: 10.1006/nimg.2001.0978

van den Bos, K., Spelberg, H., Scheepsma, A., \& de Vries, J. (1999). De klepel vorm A en B, een test voor leesvaardigheid van pseudowoorden. Verantwoording, handleiding, diagnostiek en behandeling. Lisse: Swets \& Zeitlinger.

Van der Haegen, L., \& Brysbaert, M. (2011). The mechanisms underlying the interhemispheric integration of information in foveal word recognition: Evidence for transcortical inhibition. Brain and Language, 118(3), 81-89. doi: DOI 10.1016/j.bandl.2010.03.006 
Van der Haegen, L., Cai, Q., \& Brysbaert, M. (2012). Colateralization of Broca's area and the visual word form area in left-handers: fMRI evidence. Brain and Language, 122(3), 171178. doi: 10.1016/j.bandl.2011.11.004

Van der Haegen, L., Cai, Q., Seurinck, R., \& Brysbaert, M. (2011). Further fMRI validation of the visual half field technique as an indicator of language laterality: A large-group analysis. Neuropsychologia, 49(10), 2879-2888. doi: DOI 10.1016/j.neuropsychologia.2011.06.014

Vila, P. M., \& Lieu, J. E. (2015). Asymmetric and unilateral hearing loss in children. Cell and tissue research. doi: 10.1007/s00441-015-2208-6

Vingerhoets, G., Alderweireldt, A.-S., Vandemaele, P., Cai, Q., Van der Haegen, L., Brysbaert, M., \& Achten, E. (2013). Praxis and language are linked: Evidence from co-lateralization in individuals, with atypical language dominance. Cortex, 49(1), 172-183. doi: 10.1016/j.cortex.2011.11.003

White, K. R. (2004). Early hearing detection and intervention programs: Opportunities for genetic services. American Journal of Medical Genetics Part A, 130A(1), 29-36. doi: 10.1002/ajmg.a.30048

Wilke, M., \& Lidzba, K. (2007). LI-tool: A new toolbox to assess lateralization in functional MR-data. Journal of Neuroscience Methods, 163(1), 128-136. doi: 10.1016/j.jneumeth.2007.01.026

Wu, C. M., Ng, S. H., Wang, J. J., \& Liu, T. C. (2009). Diffusion Tensor Imaging of the Subcortical Auditory Tract in Subjects with Congenital Cochlear Nerve Deficiency. American Journal of Neuroradiology, 30(9), 1773-1777. doi: 10.3174/ajnr.A1681 